\title{
ТЕОРЕТИКО-МНОЖЕСТВЕННАЯ ОПЕРАЦИЯ ВЫЧИТАНИЯ МНОГОУГОЛЬНИКОВ В ЗАДАЧАХ ТОПОЛОГИЧЕСКОГО ПРОЕКТИРОВАНИЯ
}

\author{
A. A. Бymos ${ }^{1}$ \\ ${ }^{1}$ К. т. н., доцент кафредры экономической инфрорматики учреждения образования \\ «Белорусский государственный университет информатики и радиоэлектроники», Минск, Беларусь
}

\section{Реферат}

Технологический процесс изготовления сверхбольших интегральных схем состоит из многих этапов, одним из которых является подготовка входной информации для генератора изображений фотонаборной установки. Работа посвящена решению одной из возникающих при этом частных задач - разработке метода выполнения теоретико-множественной операции вычитания над топологическими объектами типа многоугольник.

Ключевые слова: САПР СБИС, топологическое проектирование, вычислительная геометрия, теоретико-множественные операции, вычитание многоугольников.

\section{SET-THEORETIC OPERATION FOR SUBTRACTING POLYGONS IN TOPOLOGICAL DESIGN PROBLEMS}

Abstract

The technological process of manufacturing ultra-large integrated circuits consists of many stages, one of which is the preparation of input information for the image generator of the photodetector. The paper is devoted to solving one of the particular problems that arise in this case - the development of a method for performing a set-theoretic subtraction operation on topological objects of the polygon type.

Keywords: CAD VLSI, topological design, computational geometry, set-theoretic operations, subtraction of polygons

\section{Введение}

Одной из важных составных частей технологического процесса изготовления сверхбольших интегральных схем (СБИС) является подготовка с помощью систем автоматизированного проектирования (САПР) входной информации для генератора изображений фотонаборной установки. Для каждого объекта топологии создается изображение, которое составляется из отдельных областей-прямоугольников, объединение которых дает изображение всего объекта.

Создание управляющей программы для генерации изображения порождает большое число задач, многие из которых решаются методами вычислительной геометрии и оперируют обычно геометрическими объектами типа многоугольник или прямоугольник [1-11]. При решении такого рода задач часто возникает необходимость в использовании теоретико-множественных операций над многоугольниками, позволяющими находить их объединение [10], пересечение [11] и разность (вычитание). Данная статья посвящена разработке способов выполнения теоретико-множественной операции вычитания над топологическими объектами типа многоугольник.

\section{Основные понятия и определения}

Многоугольник $M$ на плоскости можно задать его границей замкнутой непересекающейся ломаной линией, состоящей из отрезков прямых, называемых сторонами многоугольника. Эту границу можно определить с помощью упорядоченного множества угловых точек или вершин многоугольника $P=\left(p_{1}, p_{2}, \ldots, p_{n}\right)$, получаемых при последовательном обходе его вдоль границы (рисунок 1). В свою очередь, угловую точку $p_{i}$, где $i \in\{1,2, \ldots, n\}$, можно задать парой $\left(x_{i}, y_{i}\right)$ декартовых координат на плоскости.

Обход границы многоугольника, а также перемещения по ее фрагментам можно выполнять в прямом или в обратном порядке. При прямом обходе отрезки границы ориентируются так, чтобы ближние к отрезку точки многоугольника всегда оставались слева, при обратном порядке - справа. Таким образом, каждой стороне $S_{i}$ многоугольника можно поставить в соответствие ориентированную прямую $v_{i}$, содержащую точки $p_{i} и p_{i+1}$. Будем считать, что она ориентирована от $p_{i}$ к $p_{i+1}$, задавая прямой порядок обхода границы многоугольника.

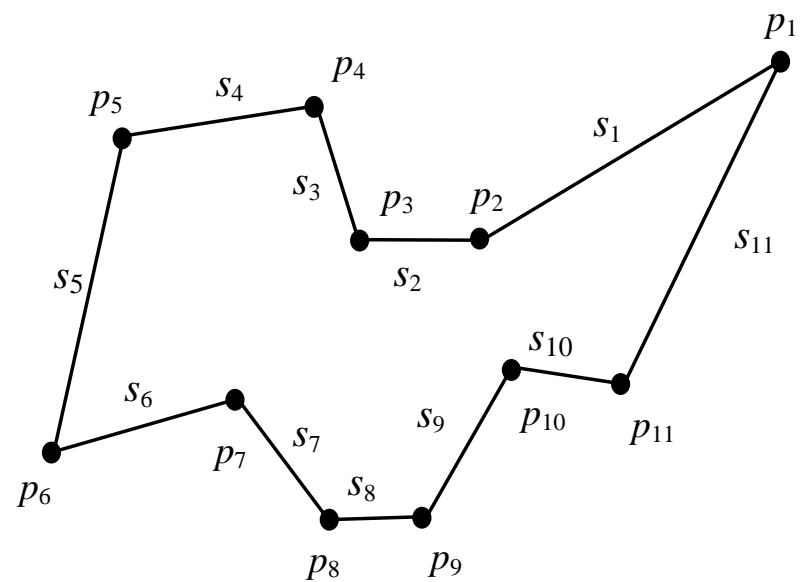

Рисунок 1 - Вершины и стороны многоугольника $M$

Вершина $p_{1}$ является начальной угловой точкой для последовательного обозначения отрезков, образующих границу многоугольника. В качестве начальной будем выбирать вершину, наиболее удаленную от начала координат.

Так как каждая пара соседних угловых точек ограничивает соответствующую сторону многоугольника, его границу можно задать также упорядоченной последовательностью сторон многоугольника $S=\left(s_{1}, s_{2}, \ldots, s_{n}\right)$, где $s_{1}=\left(p_{1}, p_{2}\right), s_{2}=\left(p_{2}, p_{3}\right), \ldots$, $s_{n}=\left(p_{n}, p_{1}\right)$.

Внутренними углами многоугольника являются углы, ограничивающие область плоскости, которую занимает многоугольник, и образованные парами его смежных сторон. Если внутренний угол меньше $180^{\circ}$, будем называть его выпуклым, а если больше $180^{\circ}-$ вогнутым (примеры выпуклого и вогнутого углов показаны на рисунках 2 и 3 в статьях [10] и [11] соответственно). 
Рассмотрим теперь два пересекающихся между собой многоугольника $M_{1}$ и $M_{2}$ (рис. 2). Результат выполнения теоретикомножественной операции вычитания или разности многоугольников $M_{1}$ и $M_{2}$ в общем случае представляет собой множество $R$ непересекающихся между собой многоугольников, каждый из которых будет содержать в себе те и только те точки плоскости, которые принадлежат многоугольнику $M_{1}$, но не принадлежат многоугольнику $M_{2}$. При этом каждая точка плоскости, принадлежащая $M_{1}$, но не принадлежащая $M_{2}$, обязательно должна содержаться в одном из элементов множества $R$. Данная теоретико-множественная операция, в отличие от теоретико-множественных операций объединения [10] и пересечения [11] многоугольников, не является коммутативной операцией и не является ассоциативной операцией.

Точки плоскости, в которых стороны многоугольников $M_{1}$ и $M_{2}$ пересекаются между собой, будут разбивать границу каждого из многоугольников на отдельные фрагменты. Рассмотрим тот из многоугольников, начальная вершина которого более удалена от начала координат. Обозначим через $\lambda_{1}, \lambda_{2}, \ldots, \lambda_{k}$ точки пересечения его сторон в порядке их встречаемости при выполнении операции прямого обхода по границе, начиная с начальной вершины. На рисунке 2 эти точки отмечены небольшими квадратами.

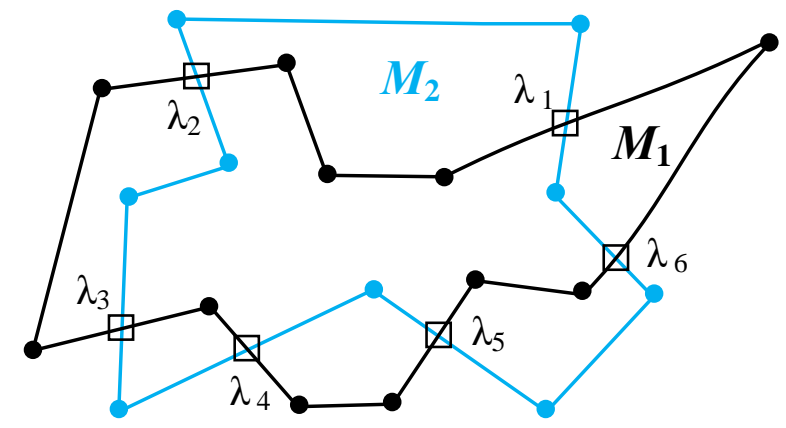

Рисунок 2 - Пересекающиеся между собой многоугольники $M_{1}$ и $M_{2}$

Фрагменты границы многоугольников $M_{1}$ и $M_{2}$, которые начинаются в точке пересечения $\lambda_{i}$ и заканчиваются в следующей по порядку точке пересечения $\lambda_{j}$, где $j=i+1$ для $i=1,2, \ldots, k-1$ и $j=1$ для $i=k$, будем называть сопряженными относительно друг друга.

Каждую пару сопряженных фрагментов, если перемещаться по одному из них в прямом направлении, а по другому - в обратном, можно рассматривать как границу многоугольника, который является частью только лишь одного из многоугольников $M_{1}$ и $M_{2}$. Такой многоугольник, граница которого из всех точек пересечения включает в себя лишь две соседние точки $\lambda_{i} и \lambda_{j}$, будем обозначать через $M\left(\lambda_{i}, \lambda_{j}\right)$ и называть в общем случае компонентом, или, при необходимости, $M_{1}$-компонентом или $M_{2}$-компонентом в зависимости от того, частью какого из многоугольников он является.

Пользуясь терминологией теории графов, будем говорить, что компонент инцидентен точке пересечения, если последняя принадлежит его границе.

Из рисунка 2 видно, что результат выполнения операции вычитания многоугольников $M_{1}$ и $M_{2}$, можно представить множеством $R=\left\{M\left(\lambda_{6} \lambda_{1}\right), M\left(\lambda_{2}, \lambda_{3}\right), M\left(\lambda_{4}, \lambda_{5}\right)\right\}$, включающим в себя из всех компонент, порождаемые точками пересечения сторон многоугольников, только лишь $M_{1}$-компоненты.

\section{Варианты пересечений сторон многоугольников между собой}

Пересечение первого типа. Это самый распространенный вариант пересечения сторон, который продемонстрирован на рисунке 2. Здесь каждая из точек пересечения является внутренней точкой для каждой из сторон, образующих пересечение. Особенностью данного варианта пересечения сторон является то, что один из смежных компонентов, инцидентных точке пересечения, есть $M_{1}$-компонент, а другой $-M_{2}$-компонент. Такие точки пересечения будем называть активными.

Пересечение второго типа. Этот вариант пересечения сторон характерен тем, что точка пересечения является внутренней точкой стороны одного многоугольника и концевой точкой двух смежных сторон другого многоугольника, т. е. совпадает с его вершиной (примеры пересечений второго типа показаны на рисунке 4 в статьях [10, 11]).

Особенностью этого варианта пересечения сторон является то, что наряду с активными точками пересечения возможны и так называемые пассивные точки пересечения, которые отличаются от активных тем, что смежные компоненты, инцидентные точке пересечения, оба являются либо $M_{1}$-компонентами, либо $\mathrm{M}_{2}$-компонентами.

Правило 1. Если обе смежные стороны многоугольника, образующие со стороной другого многоугольника пересечение второго типа, целиком содержатся в одной из полуплоскостей, расположенных слева или справа от ориентированной прямой $v_{i}$ (рисунок 3 ), то такая точка пересечения получает статус пассивной, иначе активной точки пересечения.
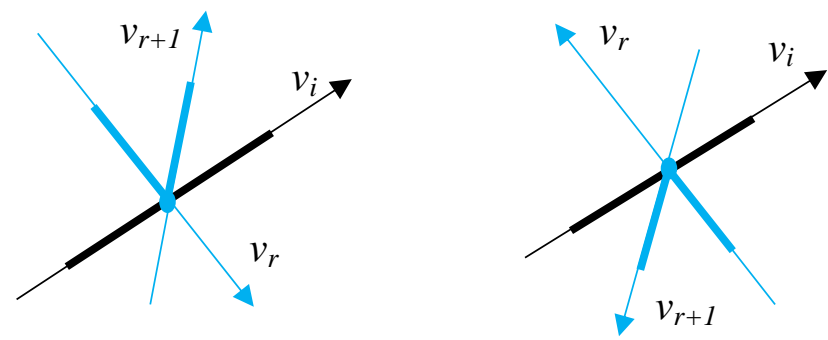

Рисунок 3 - Примеры пассивных точек пересечения второго типа

Пересечение третьего типа. Этот вариант пересечения сторон характеризуется тем, что точка пересечения является концевой точкой двух смежных сторон как в одном, так и в другом многоугольнике (примеры пересечений третьего типа показаны на рисунке 6 в статьях $[10,11])$. Данный тип пересечения также, как и пересечения второго типа, порождает как активные, так и пассивные точки пересечения.

Правило 2. Если из точки пересечения третьего типа можно провести два луча, разбивающих всю плоскость на два больших сегмента так, что смежные стороны многоугольника $M_{1}$ окажутся в одном сегменте, а смежные стороны многоугольника $M_{2}$ - в другом сегменте (рисунок 4), то такая точка пересечения получает статус пассивной, иначе - активной точки пересечения.
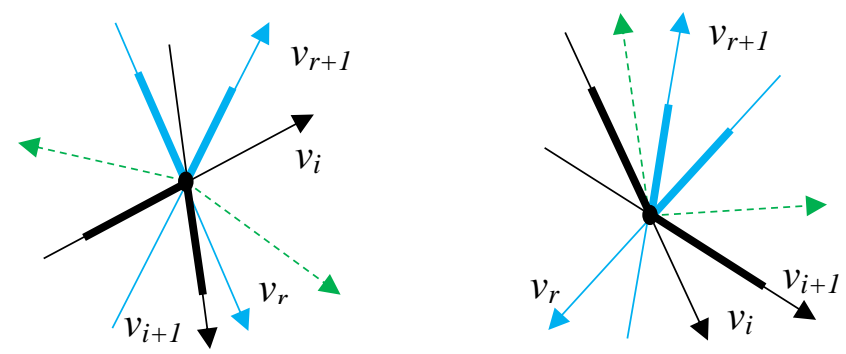

Рисунок 4 - Примеры пассивных точек пересечения третьего типа

Пересечение четвертого типа. Этот вариант пересечения сторон характерен тем, что сторона одного и сторона другого многоугольника лежат на одной прямой, при этом каждая из сторон включает в себя общий отрезок данной прямой (рисунок 5). Концевые точки такого общего отрезка рассматриваются как точки пересечения, одна из которых будет начальной, а другая - конечной в соответствии с порядком, в котором пронумерованы точки пересечения. Порождаемый этими точками компонент будем называть вырожденным, так как он представляет собой отрезок прямой. Каждая из точек пересечения четвертого типа получит статус активной или пассивной точки пересечения. Этот анализ выполняется по-разному с помощью следующих двух правил. 


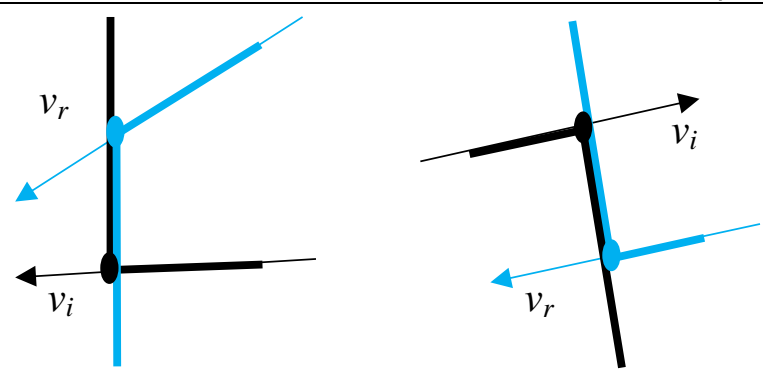

Рисунок 5 - Примеры пересечений четвертого типа

Правило 3. Используется для определения статуса конечной точки общего отрезка. При этом анализируется внутренний угол того многоугольника, который связан с этой точкой. Конечная точка получает статус пассивной, если выполняется одно из условий:

1) внутренний угол является выпуклым и принадлежит многоугольнику $M_{1}$;

2) внутренний угол является вогнутым и принадлежит многоугольнику $M_{2}$.

Если ни одно из условий не выполняется, то концевая точка получает статус активной.

Правило 4. Это правило, предназначенное для определения статуса начальной точки общего отрезка, можно использовать лишь непосредственно в процессе реализации метода вычитания многоугольников (по аналогии с поздним связыванием в объектно-ориентированном программировании), когда станет известно, $M_{1}$ - или $M_{2}$-компонентом является компонент, инцидентный начальной точке пересечения и смежный с вырожденным компонентом (общим отрезком).

Предположим теперь, что эта информация стала известной. В этом случае правило 4 применяется следующим образом. Если компонент, инцидентный начальной точке пересечения, есть $M_{1}$-компонент, то начальная точка общего отрезка получает статус активной, иначе - пассивной точки пересечения. Смысл этого правила заключается в том, чтобы любой вырожденный компонент был идентифицирован как $M_{2}$-компонент, и поэтому не мог войти в искомое решение.

\section{Метод нахождения разности многоугольников}

Предлагаемый ниже метод включает в себя два этапа. На первом этапе выполняются следующие действия:

1. Выбирается тот из многоугольников $M_{1}$ и $M_{2}$, начальная вершина которого более удалена от начала координат.
2. Выполняется обход границы многоугольника от начальной вершины в прямом направлении с последовательным нахождением всех точек пересечения $\lambda_{1}, \lambda_{2}, \ldots, \lambda_{k}$ сторон многоугольника со сторонами другого многоугольника.

3. С помощью правил 1-4 определяется статус всех точек пересечения, за исключением тех, которые являются начальными точками общих отрезков в пересечениях четвертого типа.

4. Если принадлежащий выбранному многоугольнику компонент $M\left(\lambda_{k}, \lambda_{1}\right)$ есть $M_{1}$-компонент, то он заносится в результирующее множество $R$ в качестве его первого элемента.

Второй этап состоит из $k$ - 1 шагов, на которых последовательно перебираются найденные точки пересечения $\lambda_{1}, \lambda_{2}, \ldots, \lambda_{k-1}$, и для очередной точки $\lambda_{i}$ выполняются следующие действия:

1. Если статус точки пересечения $\lambda_{i}$ не определен из-за того, что она является начальной точкой общего отрезка в пересечении четвертого типа, то этот статус уточняется в соответствии с правилом 4, поскольку на предыдущем шаге было определено, является ли компонент $M\left(\lambda_{i-1}, \lambda_{i}\right) M_{1}$ - или $M_{2}$-компонентом (для $i=1 \quad \lambda_{i-1}=\lambda_{k}$ ).

2. Рассматриваются смежные компоненты $M\left(\lambda_{i-1}, \lambda_{i}\right)$ и $M\left(\lambda_{i}, \lambda_{i+1}\right)$. Если компонент $M\left(\lambda_{i-1}, \lambda_{i}\right)$ есть $M_{1}$-компонент и $\lambda_{i}$ имеет статус активной точки пересечения или $M\left(\lambda_{i-1}, \lambda_{i}\right)$ есть $M_{2}$-компонент и $\lambda_{i}$ имеет статус пассивной точки пересечения, то компонент $M\left(\lambda_{i}, \lambda_{i+1}\right)$ идентифицируется как $M_{2}$-компонент. Если ни одно из этих двух условий не выполняется, то $M\left(\lambda_{i}, \lambda_{i+1}\right)$ идентифицируется как $M_{1}$-компонент и заносится в множество $R$.

После завершения второго этапа из всех компонент, порождаемых пересечениями сторон многоугольников $M_{1}$ и $M_{2}$, в множество $R$ будут внесены только те компоненты, которые являются $M_{1}$-компонентами. Последние в совокупности и представляют собой результат выполнения операции вычитания многоугольников $M_{1}$ и $M_{2}$.

На рисунке 6 изображены многоугольники $M_{1}$ и $M_{2}$, образующие различные типы пересечений своих сторон. Для простоты вершины многоугольников не отмечены кружками. Границы $M_{1}$-компонентов, входящих в результирующее множество $R$, отмечены пунктирной зеленой линией. Точки пересечения первого типа обозначены черными квадратами. Точки пересечения, получившие статус активных и пассивных точек пересечения, обозначены соответственно зелеными и красными квадратами.

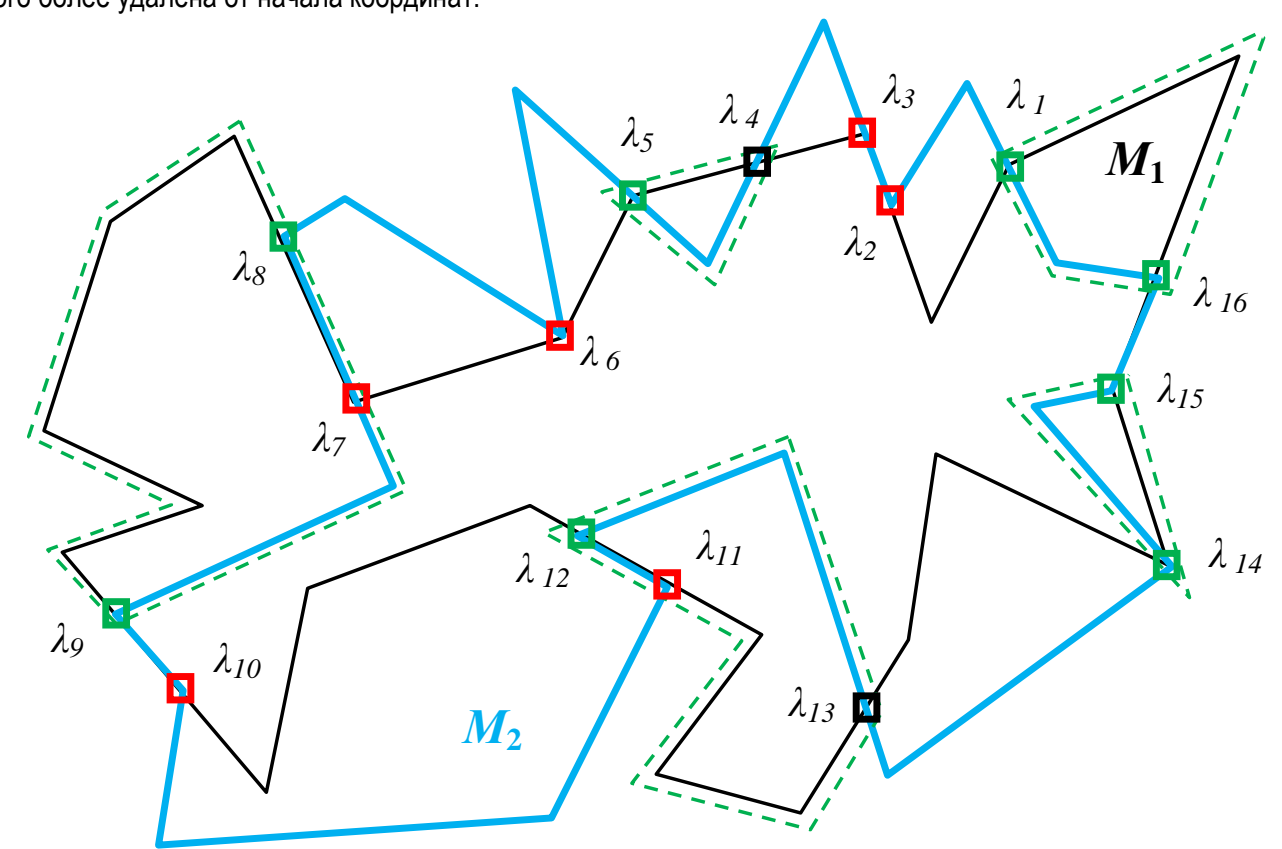

Рисунок 6 - Результат выполнения операции вычитания многоугольников $M_{1}$ и $M_{2}$ 


\section{Заключение}

Работа посвящена рассмотрению одной из частных задач, решаемых в рамках САПР СБИС, - теоретико-множественной операции вычитания топологических объектов-многоугольников. Предложен метод решения данной задачи, продуцирующий в результате один или несколько результирующих многоугольников.

Похожий, но более громоздкий метод вычитания многоугольников был ранее разработан, доведен до формы программ и опробован автором в составе автоматизированной системы подготовки информации для формирования фотошаблонов $[2,3]$.

\section{Список цитированных источников}

1. Фейнберг, В. З. Геометрические задачи машинной графики больших интегральных схем / В. 3. Фейнберг. - М. : Радио и связь, 1987. - 178 с.

2. Шестаков, Е. А. Автоматизированная система подготовки информации для формирования фотошаблонов / Е. А. Шестаков, А. А. Бутов, Т. Л. Орлова, А. А. Воронов // Искусственный интеллект. - 2008. - № 4. - С. 200-207.

3. Shestakov, E. The automated system of input data preparation for integrated circuit layout generators / E. Shestakov, A. Butov, T. Orlova, A. Voronov // Proc. Intern. Conf. "Neural Networks and Artificial Intelligence" (ICNNAl'2008). - Minsk, 2008. - P. 217-219.

4. Препарата, Ф. Вычислительная геометрия: введение : пер. с англ. Ф. Препарата, М. Шеймос. - М. : Мир, 1989. - 478 с.

5. Ласло, М. Вычислительная геометрия и компьютерная графика на С++ : пер. с англ. / М. Ласло. - М. : БИНОМ, 1997. - 304 с.

6. Шестаков, Е. А. Декомпозиция многосвязного многоугольника в множество прямоугольников / Е. А. Шестаков // Вестник БрГТУ. 2008. - № 5: Физика, математика, информатика. - С. 82-86.

7. Шестаков, Е. А. Декомпозиция многосвязного многоугольника в множество ортогональных многоугольников / Е. А. Шестаков, А. А. Воронов // Вестник БрГТУ. - 2008. - № 5: Физика, математика, информатика. - С. 87-91.

8. Бутов, А. А. Анализ корректности покрытия многосвязного многоугольника / А. А. Бутов, Е. А. Шестаков // Вестник БрГТУ. 2008. - № 5: Физика, математика, инфрорматика. - С. 57-60.

9. Бутов, А. А. Устранение избыточности в покрытии топологического объекта прямоугольниками / А. А. Бутов // Доклады БГУИР. 2017. - № 8. - С. 13-20.

10. Бутов, А. А. Теоретико-множественная операция объединения многоугольников в задачах топологического проектирования / А. А. Бутов // Информатика. - 2019. - Т.16 (№ 1). - С. 93-102.
11. Бутов, А. А. Теоретико-множественная операция пересечения топологических объектов-многоугольников на плоскости / А. А. Бутов // Доклады БГУИР. - 2019. - № 7-8 (126). - С. 58-65.

\section{References}

1. Fejnberg, V. Z. Geometricheskie zadachi mashinnoj grafiki bol'shih integral'nyh skhem / V. Z. Fejnberg. - M. : Radio i svyaz', 1987. - 178 s.

2. SHestakov, E. A. Avtomatizirovannaya sistema podgotovki informacii dlya formirovaniya fotoshablonov / E. A. SHestakov, A. A. Butov, T. L. Orlova, A. A. Voronov // Iskusstvennyj intellekt. - 2008. - № 4. S. 200-207.

3. Shestakov, E. The automated system of input data preparation for integrated circuit layout generators / E. Shestakov, A. Butov, T. Orlova, A. Voronov // Proc. Intern. Conf. "Neural Networks and Artificial Intelligence" (ICNNAl'2008). - Minsk, 2008. - P. 217-219.

4. Preparata, F. Vychislitel'naya geometriya: vvedenie : per. s angl. I F. Preparata, M. SHejmos. - M. : Mir, 1989. - $478 \mathrm{~s}$.

5. Laslo, M. Vychislitel'naya geometriya i komp'yuternaya grafika na $\mathrm{C}++$ : per. s angl. / M. Laslo. - M. : BINOM, 1997. - 304 s.

6. Shestakov, E. A. Dekompoziciya mnogosvyaznogo mnogougol'nika v mnozhestvo pryamougol'nikov / E. A. SHestakov // Vestnik BrGTU. 2008. - № 5: Fizika, matematika, informatika. - S. 82-86.

7. SHestakov, E. A. Dekompoziciya mnogosvyaznogo mnogougol'nika v mnozhestvo ortogonal'nyh mnogougol'nikov / E. A. SHestakov, A. A. Voronov // Vestnik BrGTU. - 2008. - № 5: Fizika, matematika, informatika. - S. 87-91.

8. Butov, A. A. Analiz korrektnosti pokrytiya mnogosvyaznogo mnogougol'nika / A. A. Butov, E. A. SHestakov // Vestnik BrGTU. - 2008. № 5: Fizika, matematika, informatika. - S. 57-60.

9. Butov, A. A. Ustranenie izbytochnosti v pokrytii topologicheskogo ob"ekta pryamougol'nikami / A. A. Butov // Doklady BGUIR. - 2017. № 8. - S. 13-20.

10. Butov, A. A. Teoretiko-mnozhestvennaya operaciya ob"edineniya mnogougol'nikov $\mathrm{v}$ zadachah topologicheskogo proektirovaniya I A. A. Butov // Informatika. - 2019. - T.16 (№ 1). - S. 93-102.

11. Butov, A. A. Teoretiko-mnozhestvennaya operaciya peresecheniya topologicheskih ob"ektov-mnogougol'nikov na ploskosti / A. A. Butov // Doklady BGUIR. - 2019. - № 7-8 (126). - S. 58-65.

Материал поступил в редакцию 25.02.2021 